\title{
基于深度学习的指针式仪表倾斜校正方法
}

\author{
周登科 ${ }^{1)}$, 杨颖 ${ }^{(1)}$ ，朱杰 ${ }^{2)}$ ，王库 ${ }^{2)}$ \\ 1) (中国农业大学信息与电气工程学院 北京 100083) \\ 2) (随锐科技集团股份有限公司 北京 100192) \\ (hbxtyy@126.com)
}

\begin{abstract}
摘 要: 针对仪表图像自动识别中倾斜仪表产生的读数误差，提出一种基于深度学习的圆形指针式仪表快速倾斜校 正方法，可以实现仪表图像的倾斜校正和旋转校正. 该方法利用卷积神经网络提取以表盘刻度数字为中心的关键点， 并采用最小二乘法对关键点进行椭圆拟合，结合椭圆变换理论使用透视变换对仪表图像进行第 1 次倾斜校正，再根 据一对关于仪表坚直中轴线对称的关键点计算仪表相对于水平方向的旋转角度，以拟合粗圆的几何中心为旋转中心， 旋转仪表图像实现第 2 次校正. 在变电站真实环境下采集图像数据, 验证方法性能. 实验结果表明, 该方法相对于传 统方法鲁棒性更好, 校正有效率达到 $100 \%$, 平均校正时间为 $0.45 \mathrm{~s}$, 满足实时校正需求, 识别校正后的仪表图像读数 的平均相对误差降低到 3.99\%, 平均参考误差降低到 $0.91 \%$, 充分显示该校正方法的有效性.
\end{abstract}

关键词: 仪表倾斜校正; 卷积神经网络; 最小二乘法; 椭圆拟合; 透视变换

中图法分类号: TP391.41 DOI: 10.3724/SP.J.1089.2020.18288

\section{Tilt Correction Method of Pointer Meter Based on Deep Learning}

\author{
Zhou Dengke $^{1)}$, Yang Ying ${ }^{1)^{*}}$, Zhu Jie ${ }^{2)}$, and Wang $\mathrm{Ku}^{2)}$ \\ 1) (College of Information and Electrical Engineering, China Agricultural University, Beijing 100083) \\ 2) (Suirui Group Co., Ltd., Beijing 100192)
}

\begin{abstract}
Since the tilted meter will cause reading error in the automatic recognition of meter image, a fast tilt correction method based on deep learning for circular pointer meter is proposed, which can realize the tilt correction and rotation correction of the meter image. The key points which is the center of the dial scale numbers are extracted by a convolutional neural network, and the least square method is used to fit the ellipse formed by the key points. The first tilt correction of the meter image is implemented by using perspective transform in combination with the ellipse transformation theory. Then the rotation angle of the meter relative to the horizontal direction is calculated according to a pair of symmetrical key points about the vertical central axis of the meter. The second correction is achieved by rotating the meter image with the geometric center of the fitting ellipse as the rotation center. The image data is collected in the real substation environment to verify the method performance. The experimental results show that this method is more robust than the traditional methods, with a correction efficiency of $100 \%$ and an average correction time of $0.45 \mathrm{~s}$, which can meet the requirements of real-time correction. The average relative error of reading of the meter image identified after correction is reduced to $3.99 \%$ and the average reference error is reduced to $0.91 \%$, which fully shows the effectiveness of the correction method.
\end{abstract}

收稿日期: 2020-03-27; 修回日期: 2020-07-24. 周登科(1995一), 男, 硕士研究生, 主要研究方向为图像处理、计算机视觉; 杨颖(1982-), 女, 博士, 副教授, 硕士生导师, 论文通讯作者, 主要研究方向为图像处理、视频分析、多媒体检索和模式识别; 朱杰(1988-), 女, 硕士研究生, 高级工程师, 主要研究方向为图像处理、机器视觉和计算机视觉; 王库(1955一), 男, 博士, 教授, 博士生导师, 主要研究方向为农业电气与自动化、信号与信息处理和智能机器人控制系统. 
Key words: meter tilt correction; convolutional neural network; least square method; ellipse fitting; perspective transformation

变电站中存在大量用来监测变电设备工作状 态的指针式仪表, 对这些仪表进行检测和读数识 别是一项繁重的任务. 随着智能识别系统的出现, 变电站巡检机器人上的相机可自动拍摄仪表并将 图像传人智能识别系统中进行读数, 从而代替人 工读数, 实现变电站设备的智能管理 ${ }^{[1-3]}$. 在智能 识别系统中, 指针式仪表读数的准确度很大程度 上取决于相机的拍摄角度. 当相机正对仪表拍摄 时, 采集的图像中有端正的仪表, 仪表识别较为容 易且读数准确度高. 然而, 变电站实际环境复杂, 相机自动拍摄的角度各不相同，且仪表本身存在 安装倾斜等问题，相机通常不是正对仪表的表盘 拍摄，采集到的仪表图像中的表盘往往有严重的 倾斜和旋转，同时表盘刻度也发生了比例变化，直 接对这类图像进行识别较为困难, 并且读数准确 度较低. 因此, 有必要在仪表图像智能读数前对图 像中的仪表进行倾斜校正.

在对圆形指针式仪表图像倾斜校正时，因为 待校正的仪表为圆形形状，具有高度对称性，难以 从仪表上找到可以用于校正的参考点. 因此, 目前 对于仪表图像的倾斜校正方法, 大多数是基于形 状为方形的仪表，根据仪表图像中的表盘边界线 找到透视变换点和倾斜角度, 这类仪表图像的校 正大多采用传统的车牌或者其他方形设备的校正 方法 ${ }^{[4-6]}$. 对于圆形指针式仪表，因其形状为圆形， 没有明显的线条参考物，所以无法基于仪表的边 框线条进行校正. 部分研究者通过手工标记仪表 的表盘参考点, 然后根据参考点的变化通过透视 变换校正仪表 ${ }^{[7-8]}$. 但是, 手工标记参考点的方式 难以应用在真实环境中, 对此, 部分研究者采用图 像匹配方法进行校正，即通过模板图像和待校正 的图像进行特征点匹配, 然后使用透视变换通过 匹配的点对校正仪表 ${ }^{[9-13]}$. 在图像匹配方法中，最 常用的是基于尺度不变特征变换 (scale-invariant feature transform, SIFT) ${ }^{[14]}$ 和加速稳健特征(speeded up robust features, SURF ${ }^{[15]}$ 的图像匹配方法. 图像 匹配方法可以实现较好的校正效果, 但是对于不 同类型的仪表图像需要预先采集模板图像，而图 像在匹配过程中的时效性较差. 还有部分研究者 通过图像处理技术提取仪表刻度线，根据刻度线 拟合表盘椭圆, 最后根据椭圆参数找到透视点, 以 实现仪表的倾斜校正 ${ }^{[16]}$. 这种方法的核心是提取
仪表刻度线，目前还是采用传统的图像处理方法， 速度较慢且准确度较低, 并且该方法不能同时实 现仪表的倾斜校正和旋转校正. 基于投影法的仪 表旋转角度检测和校正方法 ${ }^{[17]}$ 以及基于检测仪表 名称倾斜的方法 ${ }^{[18]}$ 通过一定的手段实现了仪表的 旋转校正，后者获得圆形指针式仪表的旋转角度， 但是均未实现仪表的透视倾斜校正.

针对目前仪表图像倾斜校正方法的不足，本 文提出一种基于深度学习的指针式仪表倾斜校正 方法, 旨在通过卷积神经网络自动提取表盘上以 刻度数字为中心的关键点, 根据关键点同时实现 仪表图像的倾斜校正和旋转校正.

\section{1 理论及算法框架}

针对现有的指针式仪表倾斜校正方法不能同 时实现仪表的倾斜校正和旋转校正, 并且在校正 过程中速度较慢、效果较差等问题, 本文提出一 种基于深度学习的指针式仪表倾斜校正方法. 该 方法分为 2 个部分: 表盘关键点提取和仪表校正. 在仪表的关键点提取中, 利用端到端的深度学习 算法 YOLOv3 提取表盘上的以刻度数字为中心的 关键点坐标. 仪表校正又分为倾斜校正和旋转校 正，首先根据提取的关键点坐标计算透视变换矩 阵，然后透视变换实现仪表的第 1 次倾斜校正; 再根据图像上的一对以表盘坚直中轴线对称的关 键点旋转图像实现仪表第 2 次旋转校正. 图 1 所 示为本文仪表图像倾斜校正的框架图.

\section{2 仪表校正方法概述}

指针式仪表图像倾斜校正的难点在于难以找 到用于校正的参考点. 本文通过粗圆拟合寻找用 于透视变换的点对坐标, 通过透视变换原理实现 仪表的第 1 次倾斜校正，再根据提取的关键点坐 标, 找到一对基于仪表中轴线对称的关键点对, 连 接这 2 个点并计算连线相对于水平方向的旋转角 度, 旋转仪表相应角度实现第 2 次旋转校正.

\section{1 基于 YOLOv3 的表盘关键点提取}

针对传统图像处理方法难以直接提取表盘关 键点, 本文提出一种基于深度学习的表盘关键点 快速提取方法. 因为仪表表盘上的各刻度数字较 

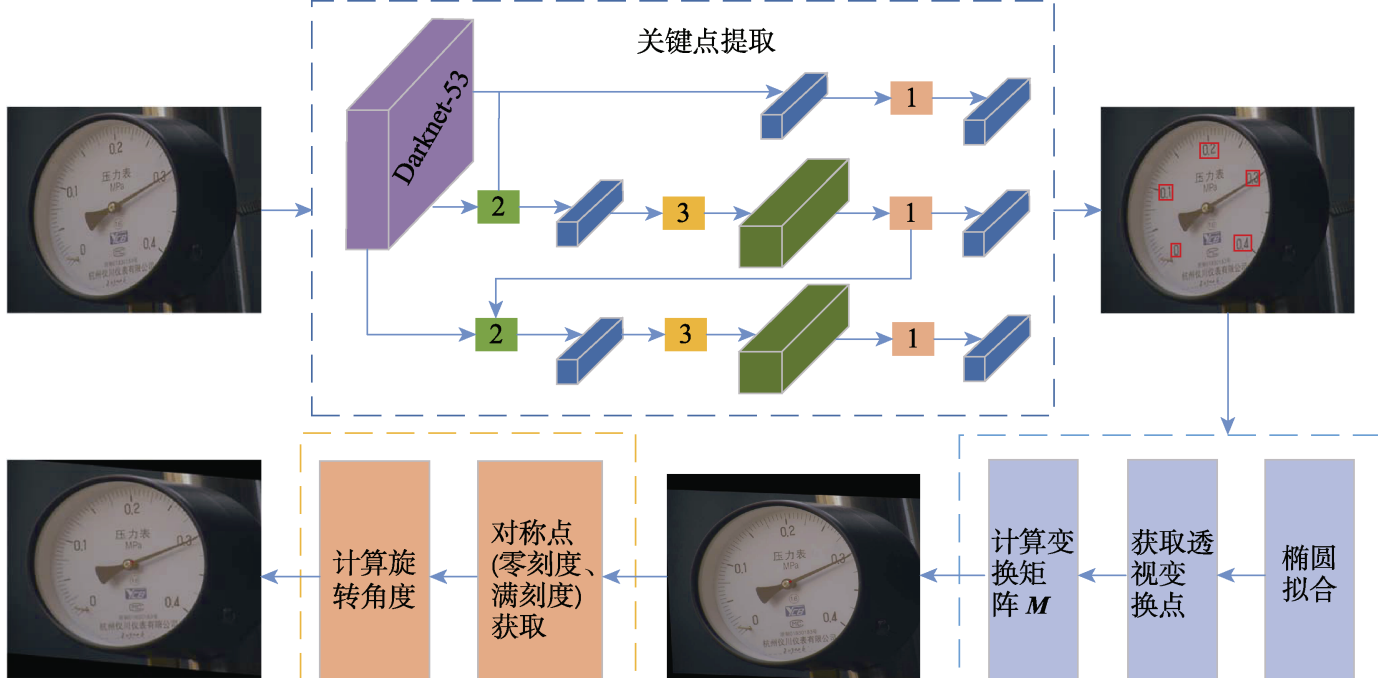

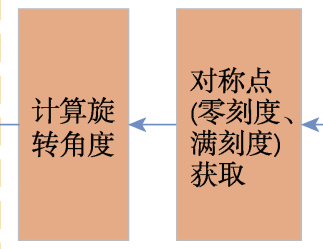

旋转校正

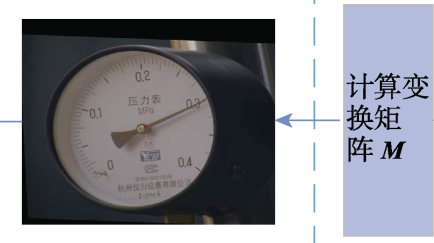

矩

$M$

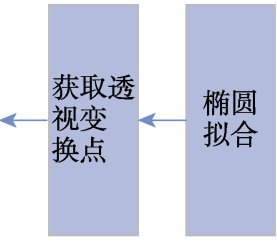

透视变换

图 1 仪表倾斜校正框架图

为明显且均匀分布, 所以本文用优异的目标检测 算法 YOLOv3 来提取表盘上的所有刻度数字, 并 以提取的刻度数字的中心作为关键点拟合椭圆.

YOLOv3 是 Redmon 等 ${ }^{[19]}$ 基于 YOLOv2 改进 的目标检测算法, 融合了 ResNet 网络、特征金字 塔(feature pyramid networks, FPN)和二元交叉熵损 失等思想. YOLOv3 以 Darknet-53 作为特征提取网 络, 该网络由 52 个卷积层和 1 个全连接层组成, 交 替使用 $3 \times 3$ 和 $1 \times 1$ 大小的卷积核进行卷积, 并输出 $13 \times 13,26 \times 26$ 和 $52 \times 52$ 的 3 种尺度特征, 在保证了 检测速度的同时，提高了小目标检测性能. 因此, 本文采用 YOLOv3 算法提取仪表关键点. 图 2 所示 为表盘关键点提取结果。

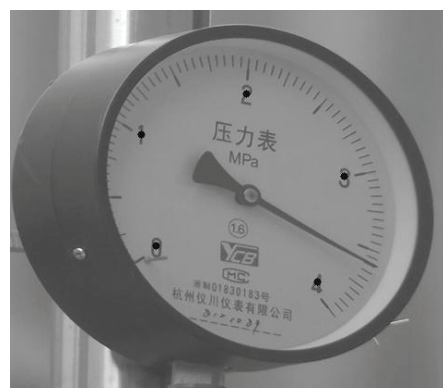

图 2 表盘关键点提取结果图

\section{2 基于透视变换的仪表倾斜校正}

仪表图像在采集中，当相机正对仪表平面时， 获得的图像为端正的仪表图像，此时表盘形状为 圆形，表盘上的各刻度数字以表盘中心为圆心均 匀分布。当相机相对仪表平面倾斜拍摄时，采集 的图像中的表盘形状为椭圆，此时仪表的各刻度
数字以椭圆的圆心为中心非均匀分布表盘椭圆 上. 因此, 只要找到这个表盘椭圆, 通过透视变 换就可以实现仪表的倾斜校正. 本文通过提取的 表盘关键点拟合椭圆，同时根据椭圆的顶点和 椭圆长短轴与椭圆的外切圆的交点作为透视变 换前后的点对，采用透视变换实现仪表的倾斜 校正.

椭圆拟合在图像处理和模式识别中有着重要 的应用 ${ }^{[20]}$. 其基本思想是用椭圆方程表示平面上点 的分布，即找到一个椭圆，使该平面上的点尽可能 地靠近椭圆. 目前, 椭圆拟合的方法很多, 最常用 的有最小二乘拟合、霍夫变换拟合和五点拟合等 ${ }^{[21]}$. 最小二乘法可以在点数未知的情况下将拟合误差最 小化, 而不同量程仪表的刻度数字的数量不同, 因 此本文采用最小二乘法拟合表盘椭圆.

平面上椭圆的一般方程可表示为

$$
A x^{2}+B x y+C y^{2}+D x+E y+1=0
$$

提取的表盘关键点坐标为 $\left(x_{i}, y_{i}\right), i=1,2, \cdots$, $N$. 其中, $N$ 代表提取的关键点个数. 对于

$$
f\left(x_{i}, y_{i}\right)=A x_{i}^{2}+B x_{i} y_{i}+C y_{i}^{2}+D x_{i}+E y_{i}+1,
$$

寻找 1 组最佳的 $(A, B, C, D, E)$ 使 $f\left(x_{i}, y_{i}\right), i=$ $1,2, \cdots, N$ 尽可能满足式(1). 因此, 基于最小二 乘法，利用 $N$ 个点构建目标函数

$$
\begin{aligned}
& F(A, B, C, D, E)= \\
& \quad \sum_{i=1}^{N}\left(A x_{i}^{2}+B x_{i} y_{i}+C y_{i}^{2}+D x_{i}+E y_{i}+1\right)^{2} .
\end{aligned}
$$

对 $(A, B, C, D, E)$ 求 $F(A, B, C, D, E)$ 的极小值. 根据最小二乘原理, 欲使 $F$ 为最小, 必有 


$$
\frac{\partial F}{\partial A}=\frac{\partial F}{\partial B}=\frac{\partial F}{\partial C}=\frac{\partial F}{\partial D}=\frac{\partial F}{\partial E}=0 .
$$

由此可得 1 个线性方程组, 然后应用求解线性方 程组的算法, 结合约束条件求得方程系数 $(A, B$, $C, D, E)$ 的值. 图 3 所示为使用最小二乘法对关 键点拟合得到的椭圆图像.

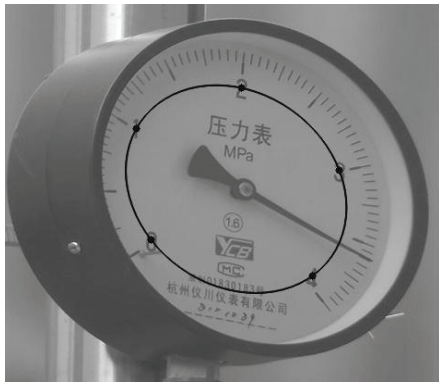

图 3 最小二乘法椭圆拟合结果

透视变换是把空间坐标系中的三维物体或对 象转变为二维图像表示的过程. 通过透视变换可 以把矩形变换成平行四边形、椭圆变成圆形等 ${ }^{[22]}$. 因此，本文通过透视变换原理将倾斜仪表图像 2 次投影为端正仪表图像。

按照透视变换原理, 若要将斜投影图像变为 垂直视角投影, 只需要找到变换前和变换后图 像的 4 个点对坐标 $\left(P_{1}, P_{2}, P_{3}, P_{4}\right)$, 映射后为 $\left(P_{5}, P_{6}, P_{7}, P_{8}\right)$, 即可联立方程求解出透视变换矩 阵; 其中 4 个点中的任意 3 个点不能在同一条直 线上. 本文透视变换点对获取方法计算过程如下： 由椭圆的一般方程可以得到椭圆的几何中心为

$$
\left\{\begin{array}{l}
x_{\mathrm{c}}=\frac{B E-2 C D}{4 A C-B^{2}} \\
y_{\mathrm{c}}=\frac{B D-2 A E}{4 A C-B^{2}}
\end{array} ;\right.
$$

椭圆短轴 $a$ 和长轴 $b$ 的半径为

$$
\left\{\begin{array}{l}
a^{2}=\frac{2\left(A x_{\mathrm{c}}^{2}+C y_{\mathrm{c}}^{2}+B x_{\mathrm{c}} y_{\mathrm{c}}-1\right)}{A+C+\left((A-C)^{2}+B^{2}\right)^{1 / 2}} \\
b^{2}=\frac{2\left(A x_{\mathrm{c}}{ }^{2}+C y_{\mathrm{c}}{ }^{2}+B x_{\mathrm{c}} y_{\mathrm{c}}-1\right)}{A+C-\left((A-C)^{2}+B^{2}\right)^{1 / 2}}
\end{array} ;\right.
$$

椭圆长轴的水平倾斜角度为

$$
\eta= \begin{cases}0, & b=0, a<c \\ \frac{1}{2} \pi, & b=0, a>c \\ \frac{1}{2} \arctan \frac{b}{a-c}, & b \neq 0, a<c \\ \frac{\pi}{2}+\frac{1}{2} \arctan \frac{b}{a-c}, & b \neq 0, a>c\end{cases}
$$

由椭圆长轴倾斜角度得到椭圆长轴的直线斜 率 $k_{1}=\tan \eta$, 椭圆短轴的直线斜率 $k_{2}=-1 / k_{1}$; 同 时联立椭圆的中心坐标, 得到椭圆的长轴和短轴 直线方程为 $y=k x+y_{\mathrm{c}}-k x_{\mathrm{c}}$. 其中, 当 $k=k_{1}$ 时, 其为长轴直线方程; $k=k_{2}$ 时, 为短轴直线方程. 由长短轴直线方程再联立栯圆的一般方程求交点, 最后得到 4 个坐标点 $\left(P_{1}, P_{2}, P_{3}, P_{4}\right)$ 即为透视变换 前的坐标点对. 由椭圆几何中心坐标和长轴半径 得到椭圆的外切圆方程为 $\left(x-x_{c}\right)^{2}+\left(y-y_{c}\right)^{2}=b^{2}$. 由椭圆外切圆方程同时联立栯圆的长短轴直线方 程求交点坐标, 最后得到透视变换后的坐标点对 $\left(P_{5}, P_{6}, P_{7}, P_{8}\right)$, 得到的映射坐标点对如图 4 所示.

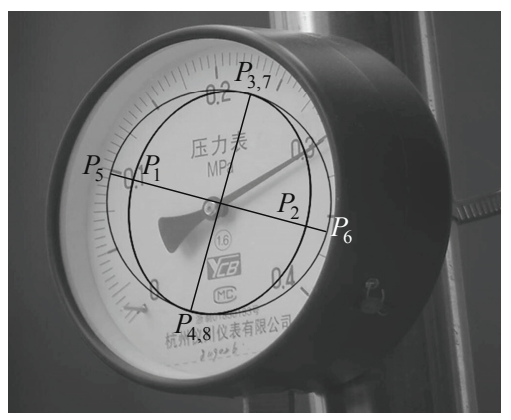

图 4 基于椭圆拟合的透视变换示意图

获得透视变换点对的坐标后, 即可求透视变 换矩阵实现仪表图像倾斜校正. 透视变换的完整 形式为

$$
\left[x^{\prime}, y^{\prime}, w^{\prime}\right]=[u, v, w]\left[\begin{array}{lll}
a_{11} & a_{12} & a_{13} \\
a_{21} & a_{22} & a_{23} \\
a_{31} & a_{32} & a_{33}
\end{array}\right]
$$

其中, $u$ 和 $v$ 是原始图像的某点坐标, 经透视变换 后得到的对应图像坐标为 $x$ 和 $y$. 将式(2)变换为 等式形式, 即

$$
\left\{\begin{array}{l}
x=\frac{x^{\prime}}{w^{\prime}}=\frac{a_{11} u+a_{21} v+a_{31}}{a_{13} u+a_{23} v+a_{33}} \\
y=\frac{y^{\prime}}{w^{\prime}}=\frac{a_{12} u+a_{22} v+a_{32}}{a_{13} u+a_{23} v+a_{33}}
\end{array}\right.
$$

将 4 组点对代人等式(3)中得到 8 个方程组, 联立 方程组可得 8 个参数, 最后获得变换矩阵 $\boldsymbol{M}$; 其 中, $a_{33}=1$. 经过透视变换校正后的仪表图像如 图 5 所示.

\section{3 基于对称性特征的仪表旋转校正}

经过透视变换完成仪表的倾斜校正，可实现 将不同比例尺度的刻度比例化. 然而, 当仪表图 像同时存在旋转情况时, 经过透视变换并不能端 
正仪表，而旋转的仪表图像增加了刻度字符识别 的难度. 同时，在仪表端正的情况下，仪表的起 始刻度和终止刻度相对于仪表的坚直中轴线对 称; 当仪表图像发生旋转时, 也增加了寻找起始 和终止刻度线的难度. 因此, 如果同时实现仪表 图像倾斜校正和旋转校正, 对于仪表的智能识别 具有重要的意义.

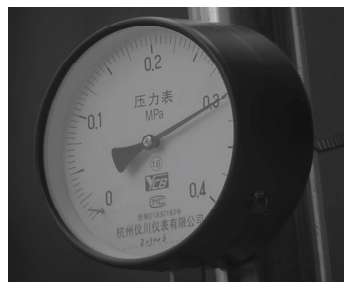

a. 原始图像

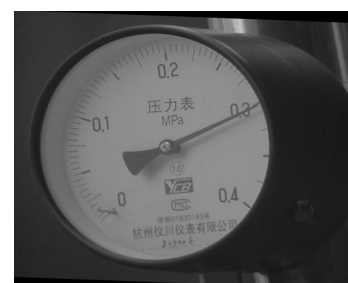

b. 透视变换校正的图像
图 5 基于透视变换的仪表校正结果示意图

通过观察仪表图像可以发现，端正的仪表图 像的所有刻度线和刻度字符都是以过表盘中心的 坚直线对称，本文定义这条线为仪表的坚直中轴 线, 如图 6a 所示. 因此, 只需要在仪表上找到一 对基于表盘坚直中轴线对称的 2 个点, 并计算连 线相对于水平方向的旋转角度, 即可完成仪表的 旋转校正.

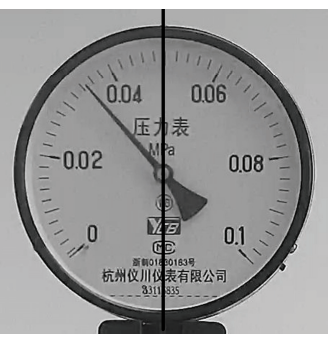

a. 仪表中轴线示意图

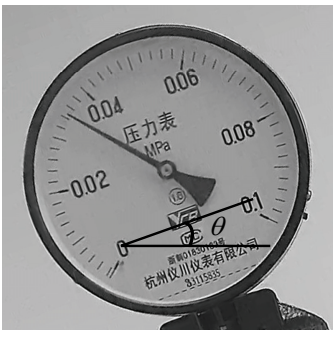

b. 仪表的旋转角度图
图 6 仪表旋转角度计算示意图

仪表的零刻度和满刻度位于刻度始末端并且 关于过表盘中心的坚直中轴线对称．因此，本文 通过寻找零刻度和满刻度对应的关键点来计算仪 表的旋转角度, 并以拟合椭圆的几何中心为旋转 中心，旋转透视变换处理后的图像实现仪表的旋 转校正.

仪表零刻度和满刻度的坐标位置可通过如下 方式获得. 设透视变换后的关键点三维坐标为 $A_{1}=$ $[X, Y, Z]^{\mathrm{T}}$, 根据透视变换原理, 变换前后的坐标点 对应关系为 $\boldsymbol{A}_{1}=\boldsymbol{M} \times \boldsymbol{B}_{1}$. 其中, $\boldsymbol{B}_{1}=[x, y, 1]^{\mathrm{T}}$ 为透 视变换前的三维坐标; $\boldsymbol{M}$ 为透视变换矩阵. 将透视 变换后的三维坐标转换为二维空间中的点坐标为 $\left(x^{\prime}, y^{\prime}\right)=\left(\frac{X}{Z}, \frac{Y}{Z}\right)$. 因此, 经过透视变换后的表盘 几何中心坐标为 $\left(x_{\mathrm{c}}^{\prime}, y_{\mathrm{c}}^{\prime}\right)$, 过椭圆中心的坚直中轴 线为 $x=x_{\mathrm{c}}^{\prime}$. 表盘关键点被坚直中轴线划分为左右 2 部分, 设左边部分的关键点坐标为 $\left(x_{i}^{\prime}, y_{i}^{\prime}\right)$. 其 中, $i=1,2, \cdots, N ; N$ 表示中轴线左边关键点的个 数. 遍历左边所有关键点, 并寻找 $y_{i}^{\prime}$ 值最大的点 即为零刻度所对应的关键点. 同理，可找到满刻度 对应的关键点位置.

获得基于表盘中轴线对称的关键点后，连接 2 点并计算连线相对于水平方向的旋转角度; 以拟 合椭圆的圆心为中心，旋转图像相应角度即可完 成仪表的旋转校正. 其中, 仪表旋转的坐标变换公 式为

$$
\left[\begin{array}{lll}
x^{\prime \prime} & y^{\prime \prime} & 1
\end{array}\right]=\left[\begin{array}{lll}
x^{\prime} & y^{\prime} & 1
\end{array}\right]\left[\begin{array}{rrr}
\cos \theta & -\sin \theta & 0 \\
\sin \theta & \cos \theta & 0 \\
0 & 0 & 1
\end{array}\right] .
$$

其中, $\left(x^{\prime}, y^{\prime}\right)$ 是旋转前的图像坐标; $\left(x^{\prime \prime}, y^{\prime \prime}\right)$ 是旋 转后的坐标值; $\theta$ 为仪表的旋转角度. 旋转后的仪 表图像如图 7 所示.

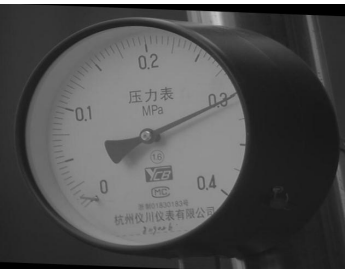

a. 透视变换后的图像

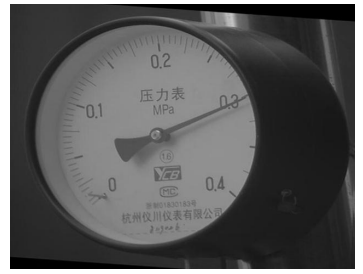

b. 旋转校正后的仪表图像
图 7 仪表旋转校正示意图

\section{3 实验结果与分析}

\section{1 基于 YOLOv3 的表盘关键点提取实验}

为了模拟仪表实际所处环境的复杂性，采用 的数据集由巡检机器人的可见光相机采集得到. 设置仪表处于不同倾斜角度、不同旋转角度和不同 光照 3 种情况, 共采集 10 类仪表, 数据集图片 5895 张; 其中训练集 5126 张, 验证集 569 张, 测 试集 200 张. 训练阶段采用动量为 0.9 的异步随机 梯度下降, 每一个 batch 包含 16 张图片, 权值的初 始学习率为 0.001 , 衰减系数设置为 0.0005 , 训练 迭代次数为 5000 次, 当迭代次数为 2000 次和 3000 次时, 分别将学习率降低为 0.00010 和 0.00001 , 使损失函数进一步收玫. 同时，训练过 
程中利用旋转和增加对比度等方法对数据集中的 图像增强和扩充. 训练过程记录模型的迭代次数 和平均损失函数之间的变换关系，结果如图 8 所 示. 从图 8 所示曲线可以看出, 随着训练次数增加, 损失值不断下降, 迭代次数到 2000 次后损失函数 趋于稳定，此时检测网络收敛.

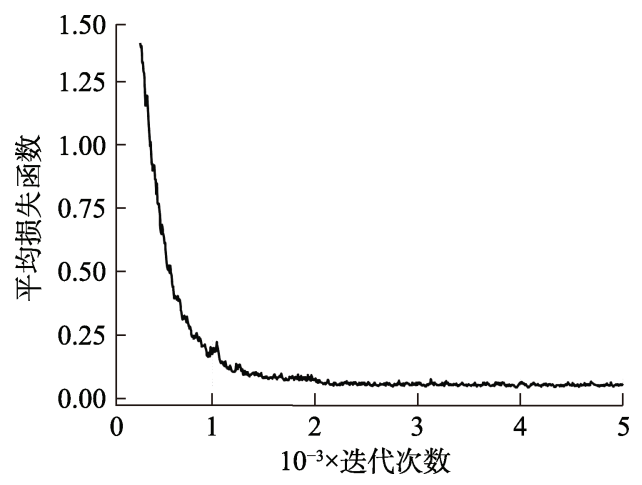

图 8 YOLOv3 模型训练中的平均损失函数曲线

本次实验的软硬件环境为 $2.90 \mathrm{GHz}$ Intel Core $^{\text {TM }}$ i5-9400 6 核 CPU; NVIDIA GeForce GTX $1660 \mathrm{Ti}(6 \mathrm{~GB})$; 内存 $8 \mathrm{~GB} ; 64$ 位 Windows 10 操作 系统; Darknet 结合 CUDA 8.0 和 CUDNN 5.1 深度 学习框架; VS2015 和 OpenCV3.4.5 开发平台.

为了准确地评估模型对表盘关键点的提取效

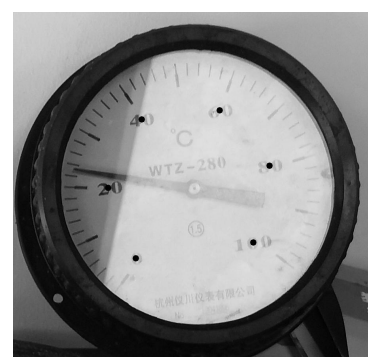

a. 反光

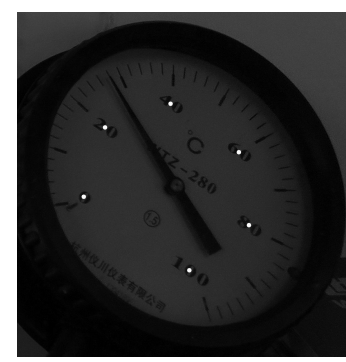

b. 背光
果, 分别计算模型对关键点提取的精准率 $P$ 和召 回率 $R$, 其计算公式分别为

$$
\left\{\begin{array}{l}
P=\frac{T_{P}}{T_{P}+F_{P}} \times 100 \% \\
R=\frac{T_{P}}{T_{P}+F_{N}} \times 100 \%
\end{array}\right.
$$

其中, $T_{P}$ 表示模型正确检测出来的目标数量; $F_{P}$ 表示模型误检的目标数量; $F_{N}$ 表示模型漏检的目 标数目.

使用训练好的 YOLOv3 模型对测试集中的 200 张图片共 1100 个关键点进行检测, 得到模型 检测的精准率为 $100 \%$, 检测的召回率为 $99.55 \%$, 统计结果充分说明了本文训练的模型对表盘关键 点提取的有效性. 关键点作为仪表校正过程中的 重要特征点, 提取的准确度将直接影响校正的准 确度, 本文对表盘关键点提取的高准确性也为仪 表的准确校正提供了保证.

为了验证本文训练模型的鲁棒性，分别对反 光、背光、刻度数字被指针遮挡和表盘模糊的仪表 图像进行关键点提取, 实验结果如图 9 所示. 从提 取结果可以看出, 本文所训练的模型对表盘关键 点提取具有较好的鲁棒性, 即使图像处于特殊情 况下，仍然可以得到非常准确的提取结果.

图 9 基于 YOLOv3 的表盘关键点提取结果

\section{2 仪表校正实验}

采用本文方法对图 10a 所示真实场景下采集 的仪表图像进行校正测试, 2 次校正结果如图 10b 和图 10c 所示. 从图 10 中可以看出, 通过本文 2 次校 正过程实现了仪表图像的倾斜校正和旋转校正.

\section{3 方法性能分析与对比}

为了验证本文倾斜校正方法对仪表读数的有 效性，对比了仪表图像在校正前后的读数、读数的 平均相对误差 $\bar{\delta}$ 和平均参考误差 $\bar{\gamma}$. 其中，仪表读 数方法使用工业中最常用的角度法 ${ }^{[23-25]}$. 该方法 通过一系列的图像处理技术获得待识别仪表图像
的指针、零刻度和满刻度位置, 然后根据指针直线 与零刻度线和满刻度线之间的角度关系得到读数. 仪表读数计算公式为

$$
R=v_{\text {max }} \times \frac{\beta}{\alpha}
$$

其中, $v_{\max }$ 为仪表最大刻度值; $\alpha$ 为零指针与最大 刻度线之间的角度; $\beta$ 为指针线与零刻度线之间 的夹角. 从式(4)可以看出, 仪表校正方法主要是 通过恢复仪表刻度之间角度比例关系，使其尽可 能地符合端正仪表图像的角度比例来达到校正效 果. 因此，采集处于不同倾斜角度下仪表图像，分 

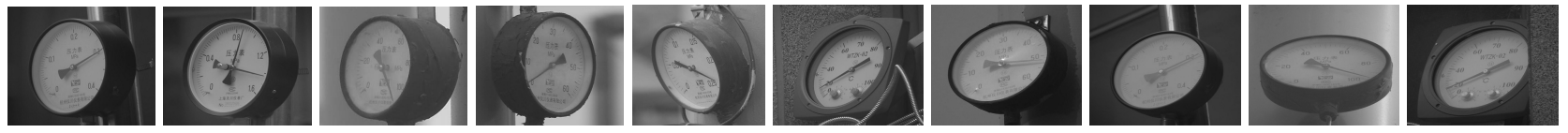

a. 原始图像
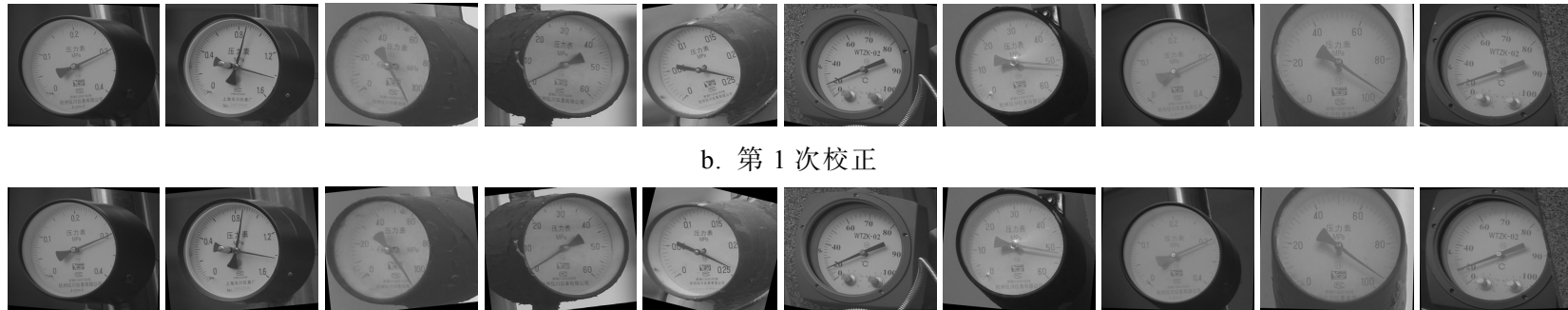

b. 第 1 次校正
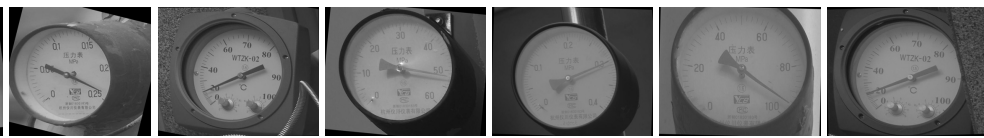

c. 第 2 次校正

图 10 仪表的校正示意图

别对比图像校正前后的指针与始末刻度线之间的 角度变化, 实验结果如表 1 所示. 其中, $\alpha^{\prime}$ 和 $\beta^{\prime}$ 是仪表处于端正情况下人为测量得到的角度; $\Delta$ 为角度比值的绝对误差, 其计算公式为

$$
\Delta=\left|\frac{\beta}{\alpha}-\frac{\beta^{\prime}}{\alpha^{\prime}}\right| \times 100 \% .
$$

从表 1 可以看出, 校正后图像的角度比值的绝 对误差明显减小, 平均绝对误差为 $2.94 \%$, 相对于 校正前的图像，误差减小了 $8.12 \%$. 充分证明校正 后的图像明显改善了仪表刻度的比例变换，使图 像的比例尺度更接近端正的仪表图像.

表 1 仪表校正前后角度

\begin{tabular}{|c|c|c|c|c|c|c|c|c|}
\hline \multirow{2}{*}{ 序号 - } & \multicolumn{2}{|c|}{ 模板图/( $\left.{ }^{\circ}\right)$} & \multicolumn{2}{|c|}{ 倾斜图/( $\left.{ }^{\circ}\right)$} & \multicolumn{2}{|c|}{ 校正图/( $\left.{ }^{\circ}\right)$} & \multicolumn{2}{|c|}{$\Delta / \%$} \\
\hline & $\alpha^{\prime}$ & $\beta^{\prime}$ & $\alpha$ & $\beta$ & $\alpha$ & $\beta$ & 倾斜图像 & 校正图像 \\
\hline 1 & 88 & 182 & 92 & 178 & 85 & 185 & 3.33 & 2.41 \\
\hline 2 & 88 & 182 & 97 & 175 & 85 & 185 & 7.08 & 2.41 \\
\hline 3 & 88 & 182 & 106 & 180 & 86 & 187 & 10.54 & 2.36 \\
\hline 4 & 88 & 182 & 120 & 185 & 82 & 188 & 16.51 & 4.73 \\
\hline 5 & 88 & 182 & 125 & 190 & 85 & 188 & 17.82 & 2.81 \\
\hline $\begin{array}{l}\text { 平均 } \\
\text { 误差 }\end{array}$ & & & & & & & 11.06 & 2.94 \\
\hline
\end{tabular}

同时，实验中使用式(4)计算仪表图像校正前 后的读数值、读数的平均相对误差 $\bar{\delta}$ 和平均参考误 差 $\bar{\gamma}$, 实验结果如表 2 所示. 其中, 仪表读数误差 计算公式为

$$
\left\{\begin{array}{l}
\bar{\delta}=\frac{\sum_{i=1}^{N} \frac{\left|R_{\text {true }}-R_{\text {test }}\right|}{R_{\text {true }}}}{N} \times 100 \% \\
\bar{\gamma}=\frac{\sum_{i=1}^{N} \frac{\left|R_{\text {true }}-R_{\text {test }}\right|}{v_{\max }}}{N} \times 100 \%
\end{array} .\right.
$$

其中, $R_{\mathrm{true}}$ 是人工记录的读数结果; $R_{\mathrm{test}}$ 是通过实
验的读数结果; $v_{\text {max }}$ 是仪表的最大刻度值; $N$ 为 实验的总数据. 图 11 所示是 10 幅不同的仪表图像 在校正前后的读数对比结果.

表 2 仪表校正前后读数和参考误差对比结果 $\%$

\begin{tabular}{cccccc}
\hline \multirow{2}{*}{ 序号 } & \multicolumn{2}{c}{ 相对误差 } & & \multicolumn{2}{c}{ 参考误差 } \\
\cline { 2 - 3 } \cline { 6 - 6 } \cline { 5 - 6 } & 校正前 & 校正后 & & 校正前 & 校正后 \\
\hline 1 & 17.97 & 12.71 & & 1.91 & 1.35 \\
3 & 35.57 & 8.33 & & 7.83 & 1.83 \\
4 & 20.17 & 3.03 & & 6.65 & 1.00 \\
4 & 17.29 & 2.83 & & 4.11 & 0.67 \\
5 & 9.42 & 3.41 & & 3.07 & 1.11 \\
6 & 2.67 & 1.17 & & 1.52 & 0.67 \\
7 & 33.63 & 4.76 & & 3.36 & 0.48 \\
8 & 3.71 & 0.23 & & 0.87 & 0.05 \\
9 & 1.53 & 3.39 & & 0.87 & 1.91 \\
10 & 29.54 & 7.64 & & 6.69 & 1.73 \\
\hline 平均误差 & 17.15 & 3.99 & 3.02 & 0.91 \\
\hline
\end{tabular}

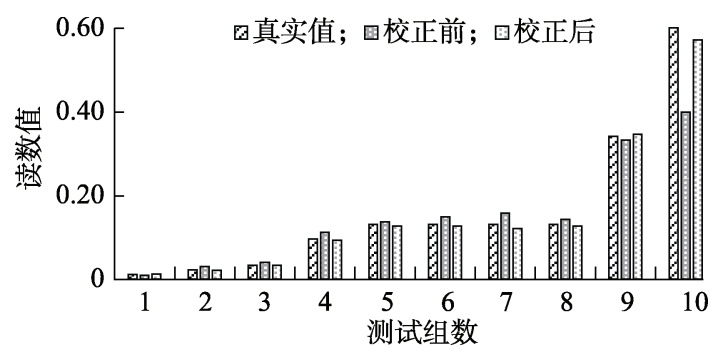

图 11 校正前后读数对比

从表 2 中可以看出，使用本文校正方法对图像 校正后再进行读数, 相对于直接识别原始图像, 读 数准确度明显提高, 读数的平均相对误差降低到 $3.99 \%$, 平均参考误差降低到 $0.91 \%$, 充分显示了 其有效性, 对于提高仪表读数的准确度具有重要 的意义. 
最后，为了验证本文校正方法相对于传统仪 表校正方法 ${ }^{[12-13]}$ 具有更好的稳定性和有效性，选 择 10 幅变电站真实环境下采集的倾斜仪表图像进 行实验校正. 校正后的图像效果如图 12 所示, 校 正效率和时间如表 3 所示; 其中有效率的统计中认
为校正后的图像相对于原图有较大的比例尺度改 善且可用于仪表读数, 则视为校正有效. 如图 12 所示, 部分图像经过透视变换后相对于原图像发 生更大的形变, 则视为校正无效, 如图 12b 所示后 7 幅图像及图 12c 所示后 5 幅图像.
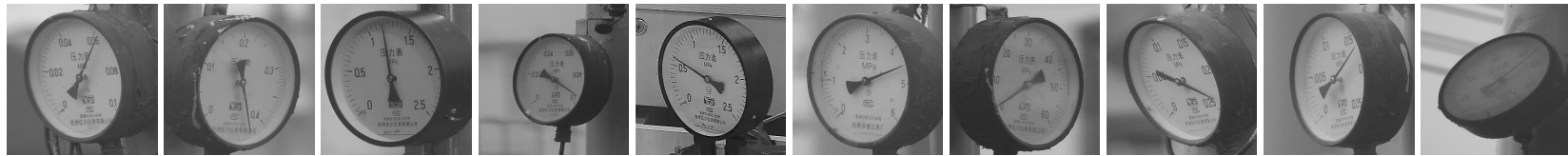

a. 原图
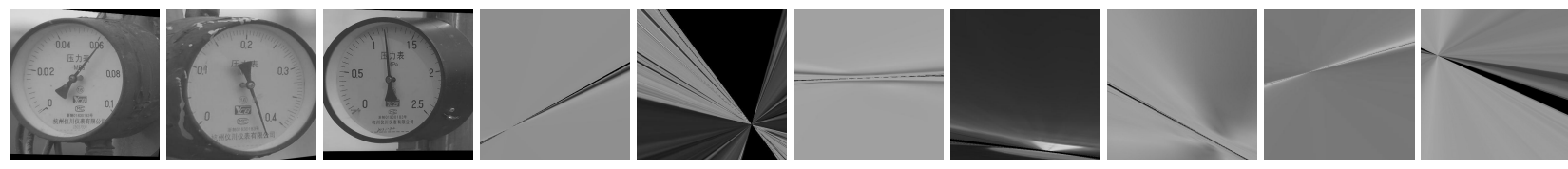

b. $\mathrm{SURF}^{[13]}$
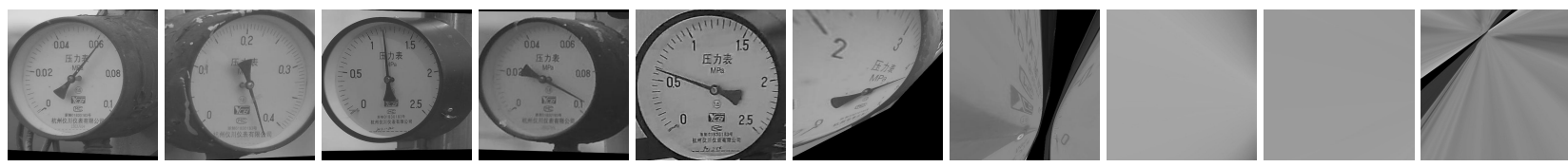

c. $\operatorname{SIFT}^{[12]}$
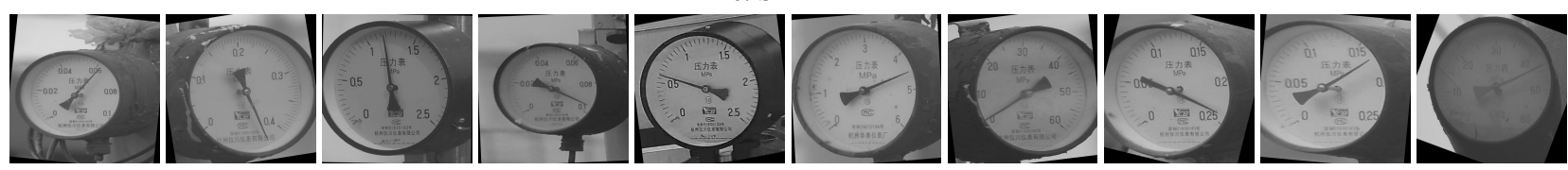

d. 本文

图 123 种方法校正结果对比

表 33 种方法校正 10 幅图像的结果对比

\begin{tabular}{lcc}
\hline \multicolumn{1}{c}{ 方法 } & 有效率 $/ \%$ & 平均时间 $/ \mathrm{s}$ \\
\hline $\mathrm{SIFT}^{[12]}$ & 50 & 0.65 \\
$\mathrm{SURF}^{[13]}$ & 30 & 0.16 \\
本文 & 100 & 0.45 \\
\hline
\end{tabular}

在表 3 对比结果中, SIFT 和 SURF 校正方法对 于模糊图像和倾斜较严重的图像校正效果较差, 因此校正有效率较低; 而本文校正方法不会受噪 声污染和倾斜程度的影响, 校正有效性可以达到 $100 \%$ ，可以满足工业复杂环境中的仪表校正需求， 同时该方法平均校正时间为 $0.45 \mathrm{~s}$, 满足实时校正 需求.

\section{4 结 语}

指针式仪表图像的倾斜校正是仪表读数识别 研究中的一项重要任务. 针对传统的图像校正方 法难以满足复杂环境中仪表的校正任务, 本文提 出一种基于深度学习的指针式仪表倾斜校正方法, 该方法通过深度卷积神经网络提取表盘上以刻度 数字为中心的关键点, 然后根据关键点信息同时
实现了仪表图像的倾斜校正和旋转校正. 实验结 果表明, 与传统校正方法相比, 本文校正方法能够 得到更好的仪表校正效果，识别校正后的仪表图 像提高了读数的准确度. 在变电站及工业环境中, 采集的仪表图像会出现各种各样的倾斜，通过本 文方法倾斜校正后再识别仪表图像，提高了读数 准确度，具有实用价值.

\section{参考文献(References):}

[1] Song Y, Deng P, Cai M, et al. The application of inspection robot in substation inspection[C] //Proceedings of the International Conference on Cloud Computing. Heidelberg: Springer, 2014: 189-196

[2] Zhang Wenjie, Xiong Qingyu, Zhang Jiaqi, et al. Pointer type meter reading recognition based on visual saliency[J]. Journal of Computer-Aided Design \& Computer Graphics, 2015, 27(12): 2282-2295(in Chinese)

(张文杰, 熊庆宇, 张家齐, 等. 基于视觉显著性的指针式仪 表读数识别算法 $[\mathrm{J}]$. 计算机辅助设计与图形学学报, 2015 , 27(12): 2282-2295)

[3] Ma Bo, Cai Weidong, Zheng Fanfan. Generating virtual samples based on prior knowledge in pointer meter recognition[J]. Journal of Computer-Aided Design \& Computer Graphics, 2019, 31(9): 1549-1557(in Chinese) 
(马波, 蔡伟东, 郑凡帆. 先验知识指导生成虚拟样本在指 针式仪表识别上的应用 $[\mathrm{J}]$. 计算机辅助设计与图形学学报, 2019, 31(9): 1549-1557)

[4] Zheng C, Wang S R, Zhang Y H, et al. A robust and automatic recognition system of analog instruments in power system by using computer vision[J]. Measurement, 2016, 92: 413-420

[5] Liu Shuang, Cui Guoguang, Liu Tonghai, et al. Character recognition of intelligent instrument based on rotation correction and sliding window location[J]. Electrical Measurement \& Instrumentation, 2013, 50(6): 20-23(in Chinese) (刘爽，崔国光，刘同海，等. 基于旋转校正和滑动窗口定位 的智能仪表字符识别 [J]. 电测与仪表, 2013, 50(6): 20-23)

[6] Wang Minqian, Lu Shufang, Zhang Yuanming, et al. A fast correction method for vehicle license plate based on local projection variance [J]. Computer Measurement \& Control, 2019, 27(1): 228-232(in Chinese)

(汪敏倩, 卢书芳, 张元鸣，等. 基于局部投影方差的快速车 牌校正方法 [J]. 计算机测量与控制, 2019, 27(1): 228-232)

[7] Huo Fengcai, Wang Di, Li Zhengzhang. Improved recognition method of industrial linear pointer meter[J]. Journal of Jilin University: Information Science Edition, 2018, 36(4): 423-429(in Chinese)

(霍凤财, 王迪，李政璋. 工业线性指针仪表识别改进方法 [J]. 吉林大学学报: 信息科学版, 2018, 36(4): 423-429)

[8] Sun Huiyuan, Yang Xiaocheng, Jiang Mingfeng, et al. Pointer instrument identification by using two-dimensional code matching[J]. Computer Systems \& Applications, 2019, 28(3): 208-214(in Chinese)

(孙慧媛, 杨晓城, 蒋明峰, 等. 基于二维码匹配的指针式仪 表读数识别方法 [J]. 计算机系统应用, 2019, 28(3): 208-214)

[9] Yang Zhijuan, Yuan Zongheng, Qiao Yu, et al. Research of intelligent recognition method of pointer instrument based on image processing[J]. Computer Measurement \& Control, 2015, 23(5): 1717-1720(in Chinese)

(杨志娟，袁纵横，乔宇，等. 基于图像处理的指针式仪表智 能识别方法研究 [J]. 计算机测量与控制, 2015, 23(5): 1717-1720)

[10] Fang Hua, Jiang Tao, Li Hongyu, et al. Double pointers meter recognition algorithm for equipment inspection $\operatorname{robot}[\mathrm{J}]$. Shandong Electric Power, 2013, 40(3): 9-13+69(in Chinese) (房桦, 蒋涛, 李红玉, 等.一种适用于智能变电站巡检机器 人的双针仪表读数的识别算法 $[\mathrm{J}]$. 山东电力技术, 2013 , 40(3): 9-13+69)

[11] Huang Yan, Li Wensheng, Mai Xiaoming, et al. Intelligent recognition method for substation pointer meter images based on one-dimensional measuring line mapping $[\mathrm{J}]$. Guangdong Electric Power, 2018, 31(12): 80-85(in Chinese) (黄炎，李文胜，麦晓明，等. 基于一维测量线映射的变电站 指针仪表智能识读方法 [J]. 广东电力, 2018, 31(12): 80-85)

[12] Xu Zunyi, Han Shaochao. Pointer instrument reading recognition based on camera calibration and maximum connected region algorithm[J]. Modern Electronics Technique, 2019, 42(9): 46-50(in Chinese)

(徐遵义, 韩绍超. 基于相机标定和最大连通区域算法的指 针式仪表读数识别 [J]. 现代电子技术, 2019, 42(9): 46-50)

[13] Gong Fangchao, Wang Shuohe, Zhou Qibin, et al. Location and readout identification of instrument in transformer substation based on SURF and FLANN algorithm[J]. Journal of Shijiazhuang Tiedao University: Natural Science Edition, 2020, 33(1): 110-115(in Chinese)

(巩方超，王硕禾，周启斌，等. 基于 SURF 和 FLANN 算法 的变电所仪表定位与读数识别 $[\mathrm{J}]$. 石家庄铁道大学学报: 自然科学版, 2020, 33(1): 110-115)

[14] Lowe D G. Distinctive image features from scale-invariant keypoints[J]. International Journal of Computer Vision, 2004, 60(2): 91-110

[15] Bay H, Tuytelaars T, van Gool L. SURF: speeded up robust features[C] //Proceedings of European Conferene on Computer Vision.Heidelberg: Springer, 2006: 404-417

[16] Liu Y, Liu J, Ke Y C. A detection and recognition system of pointer meters in substations based on computer vision[J]. Measurement, 2020, 152:107333

[17] Wang Xin, Sun Peng. Automatic identification method of the pointer instrument in intelligent substation[J]. Electrical Engineering, 2016, 17(5): 7-10(in Chinese)

(王欣, 孙鹏. 智能变电站中指针式仪表图像自动识别方法 [J]. 电气技术, 2016, 17(5): 7-10)

[18] Qiu Jinhong, Wang Renhuang, Wang Zhimin, et al. A skew detection method for circular-pointer instrument[J]. Automation \& Information Engineering, 2013, 34(4): 40-44(in Chinese) (邱金红，汪仁煌，汪志敏，等.一种圆形指针式仪表倾斜检 测方法 [J]. 自动化与信息工程, 2013, 34(4): 40-44)

[19] Redmon J, Farhadi A. YOLOv3: an incremental improvement[OL]. [2020-03-27]. https://arxiv.org/abs/1804.02767

[20] Ben X Y, Meng W X, Yan R. Dual-ellipse fitting approach for robust gait periodicity detection[J]. Neurocomputing, 2012, 79: 173-178

[21] Yuan Fu. A method of correcting the pointer reading of deflection pointer instrument $[\mathrm{C}] / /$ Proceedings of the Chinese Automation Congress. Los Alamitos: IEEE Computer Society Press, 2017: 5517-5520

[22] Guo Jiajie, Wang Yongtian, Chen Jing. A robust outdoor building image rectification method[J]. Journal of Computer-Aided Design \& Computer Graphics, 2014, 26(7): 1084-1091(in Chinese)

(国佳杰, 王涌天, 陈靖. 一种鲁棒的户外建筑物图像校正 方法 [J]. 计算机辅助设计与图形学学报, 2014, 26(7): 1084-1091)

[23] Gao J W, Xie H T, Zuo L, et al. A robust pointer meter reading recognition method for substation inspection $\operatorname{robot}[\mathrm{C}]$ //Proceedings of the International Conference on Robotics and Automation Sciences. Los Alamitos: IEEE Computer Society Press, 2017: 43-47

[24] Yang Shijie, Zhang Ping. A method for reading recognition of index-instrument[J]. Microcomputer \& Its Applications, 2017, 36(17): 59-61(in Chinese) (杨世杰, 张平. 一种指针式仪表的识别方法 [J]. 微型机与 应用, 2017, 36(17): 59-61)

[25] Shi Ying, Xia Chunhua, Hu Linna, et al. Intelligent recognition method for reading of pointer instrument based on machine vision[J]. Transducer and Microsystem Technologies, 2017, 36(11): 47-49+52(in Chinese) (施漟, 夏春华, 胡琳娜, 等. 指针式仪表读数的机器视觉智 能识别方法[J]. 传感器与微系统, 2017, 36(11): 47-49+52) 